\title{
Arabic version of the visual vertigo analogue scale for assessment visual vertigo syndrome
}

\author{
Original \\ Hossam Sanyelbhaa Talaat ${ }^{1}$, Ahmed Mahmoud Zein El Abedein ${ }^{1}$ and Reham Elhussieny \\ Article \\ $\mathrm{Ali}^{2}$ \\ ${ }^{1}$ Department of Otolaryngology, Faculty of Medicine, Menoufia University, \\ ${ }^{2}$ Department of Otolaryngology, Almenshawy Hospital, Tanta, Egypt
}

\begin{abstract}
Objectives: To standardize the Arabic language version of the visual vertigo analogue scale (VVAS) and to determine its reliability and validity. It is a simple and supplemental kind of assessments of visual vertigo syndrome.

Methods: The Arabic VVAS (AVVAS) was developed using the cross-cultural adaptation guidelines. The scale was tested by 70 patients with visual vertigo (VV) (patient group) and 100 normal subjects (control group). Participants' responses were statistically analyzed for internal consistency between both groups. Repeatability was calculated using Spearman correlation on a subgroup of 30 control subjects and $40 \mathrm{VV}$ patients after 1 week. Receiver operating characteristic curve was performed in order to select the optimal cut-off level.

Results: showed a significantly good internal consistency (Cronbach's alpha $=0.83$ for patients group, and 0.73 for controls). A significant difference was found in AVVAS scores between both groups ( $p=<0.001)$. Test- retest reproducibility confirmed reliability of AVVAS. The AVVAS total scores of patients with VV were significantly different than controls. The optimal cut-off score for AVVAS was 17 (sensitivity $80 \%$, specificity $82 \%$ ).

Conclusion: The AVVAS can be considered a reliable, valid and helpful screening tool to quantify self- perceived handicapped that result from visual vertigo.
\end{abstract}

Key Words: Validity, visual vertigo, visual vertigo analogue scale

Received: $22^{\text {th }}$ February 2019, Accepted: $21^{\text {th }}$ June 2019

Corresponding Author: Ahmed Mahmoud Zein El Abedein, M.D, Department of Otolaryngology, Faculty of Medicine, Menoufia University, Egypt, Tel.: +2 01090027979, E-mail: am_zein14@yahoo.com

ISSN: 2090-0740, November 2019 Vol.20, No.3

\section{INTRODUCTION}

Visual vertigo (VV) is a condition in which there is a worsening of vestibular symptoms in certain visual environments ${ }^{[1]}$. It has been variously termed as visual vestibular mismatch ${ }^{[2,16]}$; space and motion discomfort ${ }^{[3]}$ and visually- induced dizziness ${ }^{[4]}$. Pathophysiology of this syndrome is still poorly understood ${ }^{[5]}$. Patients often report a worsening of dizziness in moving visual backgrounds. They may experience severe panic symptoms including sweating and spinning sensation ${ }^{[6]}$. Symptoms occur in the latent phase after an acute vestibular insult ${ }^{[7-9]}$. Clinical tests are helpful in the diagnosis of vestibular dysfunction; however it is inadequate for assessing dizziness effects on the patient life or treatment progress ${ }^{[10]}$. Psychometric tool such as questionnaires seem to be an alternative method $^{[11]}$. Several balance measures have been translated into the Arabic for use with adults with vestibular disorders including the Dizziness Handicap Inventory (DHI) ${ }^{[12]}$, Visual Vestibular Mismatch Questionnaire (VVMQ) ${ }^{[13]}$, and the Situational Characteristic Questionnaire (SCQ) ${ }^{[14]}$. The Visual Vertigo Analogue Scale (VVAS) may be helpful in providing a quantitative evaluation scale of $\mathrm{VV}^{[15]}$. This scale has not been translated into the Arabic language. The goal of this study was to determine if the newly translated Arabic version of the VVAS (AVVAS) is valid for use in persons with visual vertigo symptoms that speak Arabic.

\section{MATERIALS AND METHODS}

\section{Developmental of the AVVAS}

Translation of the original 9- items VVAS was done according to the cross-cultural adaptation guidelines. The English version of VVAS was forward translated from English language into the modern-standard Arabic language by 2 bilingual audio-vestibular specialists. The translated AVVAS was re-translated into an English version by 2 independent bilingual subjects who were unaware of the original version of the VVAS. The committee of the 4 translators reviewed the forward and backward translations, and the pre-final AVVAS was created. To assess the completeness of the AVVAS items, the final AVVAS was tested on a separate group of 15 patients who fulfilled the eligibility criteria and represented a group of people with VV. After reviewing the feedback of the tested group, we found that all items of the adapted VVAS were acceptable 
to all participants. The translated Arabic version of VVAS was finalized and administered to the group of patients with VV handicap and control subjects. The authors asked the Participants to complete the Arabic VVMQ (AVVMQ) ${ }^{[13]}$ and the AVVAS during their first clinical visit. It took 5-10 minutes to complete the 2 forms and require no special training to administer. After one week, the authors re-requested 50 subjects ( 20 controls, $30 \mathrm{VV}$ patients) who attended the follow-up at audio- vestibular unit and 20 of participants were administered the scale by telephone to re- rate the intensity of dizziness they experience in each situation of the AVVAS to check for test-retest reliability.

\section{Participants:}

170 participants were recruited from the audio-vestibular medicine unit, Menoufia University, Egypt in the period from September 2017 and October 2018. 70 patients with VV (30 males and 40 females), and 100 healthy subjects (51 male, 49 female) were enrolled in the study on a first visit. Inclusion criteria were (a) aged18- 65 years; (b) ability to understand the Arabic language both orally and in writing; (c) The control group was the healthy accompanied family members of the patients, free from any vestibular/balance complaints and with no history of hearing or neurological disorder; (d) VV individuals were diagnosed according to the AVVMQ, they included if they had $\left(3,4,5\right.$ or 6 positive answers) of the AVVMQ ${ }^{[13]}$ .Exclusion criteria were (a) medications that may have affected balance or cerebral blood supply; (b) Psychiatric disorder, cognitive impairment, musculoskeletal or neurological motor disorders; (c) binocular visual acuity; (d) glaucoma. The study was approved by audio-vestibular unit, Otolaryngology Department, faculty of medicine, Menoufia University, Egypt. Written informed consent was obtained from all the participants of this study before filling the AVVAS, phone numbers were also obtained.

\section{The Visual Vertigo Analogue Scale (VVAS)}

The VVAS is a 9-item questionnaire that can be completed by the patient to assess their intensity of $\mathrm{VV}$ in challenging situations of visual motions that typically provoke dizziness; the individuals marked a vertical line on 100 millimeters straight line ${ }^{[15]}$.The 9 scales to be summarized, 2 final scores were calculated: VVAS positive and VVAS severity. The persons were classified as VVAS positive if 2 or more items were rated $(1,2,3,4,5,6,7,8$, 9, or 10) (VVAS positive answers). VV severity score of zero (no VV symptoms), and $100(100 \% \text { would indicate severe VV })^{[15]}$.

\section{Statistical analysis}

The Statistical Package of Social Science (SPSS version 22) was used for data analysis. A $p$-values $<0.005$ was considered to be statistically significant. The VV and control groups were compared with respect to sex, and age. Quantitative variables were presented as mean and standard deviation (SD). Nonparametric variables were compared between the 2 groups using Mann-Whitney test. The reliability of the AVVAS using Cronbach's alpha coefficient (internal consistency) was evaluated. Coefficient value ranges from $0-1$, with 1 indicating perfect. Internal consistency for individual factors on the AVVAS was judged to be satisfactory if value $>0.70$, good if value $>0.80$. Coefficient was also calculated for each deleted item to establish how each item affected the reliability of the instrument. Test- retest correlation was assessed by calculating Spearman non-parametric correlational coefficients. Correlations were judged to be significant at $p<0.05$ and magnitude of significant coefficients was evaluated as follows: small (0.1-0.29), mild (0.3-0.5), moderate (0.5-0.7), and strong (greater than 0.7). Receiver operating curves (ROC) assessed discriminant validity to calculate the AVVAS's sensitivity and specificity in discriminating normal versus abnormal VV symptoms. Area under the curve (ROC) values of 0.7 and higher would be considered strong predictors. Positive and negative predictive values were also performed.

\section{RESULTS}

\section{Subjects}

No significant difference noted in the age distribution (VV mean $45.6 \pm 11.9$ with range of $(18-63$ years $)$, controls mean $42.4 \pm 9.87$ with range $(19-63$ years $)$ between the patient group and the control participants. The controls included $40(570.1 \%)$ being female and $30(42.9 \%)$ being male while VV group 51 (51\%) being males and 49 (49\%) being females. There was near matching of the 2 groups as regards sex using Chi squared test $(P$ value $>0.05)$. The mean $( \pm \mathrm{SD})$ total score of the AVVAS was $35.5( \pm 16.8)$ in the VV group, and $10.9( \pm 12.1)$ in the controls. The total Arabic VVAS score were found to be significantly higher in the VV group than in the controls. Wilcoxon-Mann Whitney test showed statistically significant difference between the 2 groups ( $P$ value $<0.001)$.

\section{Reliability of the AVVAS}

Cronbach's alpha coefficient was estimated at 0.72 and 0.83 respectively for the controls and VV group. Item-tototal correlation coefficients revealed good associations between the AVVAS individual items and its total score (Table 1). There was a positive correlation between total sores of the AVVAS in the first and second visit in the $\mathrm{VV}$ and controls ( $p$ value $<0.001$ ). For VV group (first visit: the total score mean $=35.5$; $\mathrm{SD}=16.8$; second visit: mean $=34.5$., $\mathrm{SD}=17.1$, Spearman's correlation $(\mathrm{r})=$ $0.978^{*}$ ), in controls (first visit: the total score mean $=$ 10.9; $\mathrm{SD}=12.11$; second visit: mean $=10.6 ., \mathrm{SD}=11.9$, Spearman's correlation $\left.(r)=0.976^{*}\right)($ Table 2,3$)$. 
Table 1: The internal consistency of the Arabic VVAS in the control and VV group

\begin{tabular}{lcccc}
\hline & \multicolumn{2}{c}{ Control group } & \multicolumn{2}{c}{ VV group } \\
\cline { 2 - 5 } & CI-TC & $\alpha$ if item deleted & CI-TC & $\alpha$ if item deleted \\
\hline Scale 1 & 0.154 & 0.735 & 0.518 & 0.819 \\
Scale 2 & 0.424 & 0.701 & 0.448 & 0.824 \\
Scale 3 & 0.432 & 0.700 & 0.487 & 0.818 \\
Scale 4 & 0.309 & 0.720 & 0.654 & 0.800 \\
Scale 5 & 0.327 & 0.719 & 0.418 & 0.827 \\
Scale 6 & 0.599 & 0.663 & 0.706 & 0.791 \\
Scale 7 & 0.596 & 0.666 & 0.722 & 0.797 \\
Scale 8 & 0.425 & 0.701 & 0.376 & 0.836 \\
Scale 9 & 0.307 & 0.721 & 0.655 & 0.798 \\
Total score & Cronbach's Alpha & 0.729 & Cronbach's Alpha & 0.830 \\
\hline
\end{tabular}

CI-TC Corrected Item-Total Correlation; $\alpha$ : Cronbach's alpha; VV: visual vertigo; VVAS: visual vertigo analogue scale.

Table 2: The internal consistency of the Arabic VVAS in the control and VV group

\begin{tabular}{llll}
\hline Scale & First visit mean \pm SD & Second visit mean \pm SD & Spearman's correlation $(r)$ \\
\hline 1 & $0.40 \pm 1.27$ & $0.41 \pm 1.30$ & $0.322^{*}$ \\
2 & $1.90 \pm 2.28$ & $1.89 \pm 2.22$ & $0.968^{*}$ \\
3 & $1.21 \pm 1.97$ & $1.30 \pm 1.94$ & $0.856^{*}$ \\
4 & $1.49 \pm 2.19$ & $1.45 \pm 2.15$ & $0.998^{*}$ \\
5 & $0.91 \pm 1.91$ & $0.85 \pm 1.74$ & $0.999^{*}$ \\
6 & $1.24 \pm 2.24$ & $1.23 \pm 2.20$ & $0.995^{*}$ \\
7 & $0.77 \pm 1.64$ & $0.74 \pm 1.56$ & $0.999^{*}$ \\
8 & $1.97 \pm 2.51$ & $1.95 \pm 2.46$ & $0.999^{*}$ \\
9 & $1.07 \pm 2.26$ & $1.02 \pm 2.07$ & $0.998^{*}$ \\
Total score & $10.9 \pm 12.1$ & $10.6 \pm 11.9$ & $0.976^{*}$ \\
\hline
\end{tabular}

VVAS: visual vertigo analogue scale; SD: Standard deviation * Significant

Table 3: Test-retest reliability of the Arabic VVAS in the VV group

\begin{tabular}{llll}
\hline Scale & First visit mean \pm SD & Second visit mean \pm SD & Spearman's correlation (r) \\
\hline 1 & $0.57 \pm 1.12$ & $1.08 \pm 1.42$ & $0.373^{*}$ \\
2 & $6.02 \pm 3.62$ & $6.40 \pm 3.37$ & $0.969 *$ \\
3 & $4.12 \pm 3.23$ & $3.45 \pm 3.04$ & $0.687^{*}$ \\
4 & $5.07 \pm 3.03$ & $4.70 \pm 3.09$ & $0.994^{*}$ \\
5 & $3.74 \pm 3.47$ & $3.64 \pm 3.25$ & $0.982^{*}$ \\
6 & $4.50 \pm 3.99$ & $4.29 \pm 4.04$ & $0.994 *$ \\
7 & $4.17 \pm 3.65$ & $3.94 \pm 3.77$ & $0.996^{*}$ \\
9 & $3.64 \pm 3.79$ & $3.75 \pm 4.05$ & $0.997 *$ \\
Total score & $2.90 \pm 3.21$ & $3.24 \pm 3.28$ & $0.994 *$ \\
\hline
\end{tabular}

VVAS: visual vertigo analogue scale; SD: Standard deviation $\quad *$ Significant 


\section{Receiver-Operating Characteristic (ROC) curve}

ROC curve demonstrated the best psychometric properties for a cut off score of 17 with a sensitivity of $80 \%$, specificity of $82 \%$, a positive predictive value (PPV)
$76 \%$, a negative predictive value (NPV) of $85 \%$ and accuracy $81 \%$ (Table 4 ). The scale' ability to discriminate between the VV and control groups was good as indicated by the area under the ROC curve (Figure 1).

Table 4: Sensitivity and specificity of the Arabic form of visual vertigo analogue scale in diagnosis of visual vertigo

\begin{tabular}{lllllll}
\hline AUC & Cut off point & Sensitivity (\%) & Specificity (\%) & Positive predictive value (\%) & Negative predictive value (\%) & Accuracy (\%) \\
\hline 0.897 & 17.0 & 0.80 & 0.82 & 0.76 & 0.85 & 0.81 \\
\hline
\end{tabular}

- $\quad \mathrm{AUC}=$ Area under the receiver operating characteristic curve.

- Accuracy: The percentage of patients correctly identified as diseased and non-diseased.

- Sensitivity (positivity in disease) refers to the Proportions of diseased patients who are reported as positive i.e. positive in disease

- $\quad$ Specificity refers to the Proportions of diseased free patients who are reported as negative i.e., negative in health.

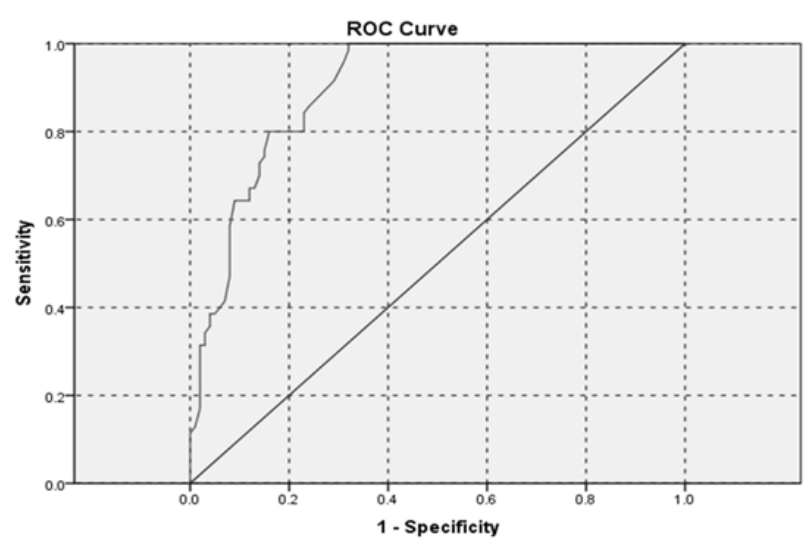

Fig. 1: Receiver operating characteristic curve (ROC) illustrating the ability of the Arabic visual vertigo analogue scale to discriminate between controls and VV patients

- The ROC curve: It is a graphic representation of the relationship between sensitivity and specificity at different cut-off points for a diagnostic test.

\section{DISCUSSION}

The main scope of this study was to provide the researchers a reliable tool to facilitate $\mathrm{VV}$ assessment among the Arab population. The AVVAS was tested in Patients and in healthy control subjects. The small time is needed to fill in the tool. In our work, the mean age of VV patients was $45.6 \pm 9.87$ with range $(18-65$ years $)$. These results were similar to those in the study by Talaat et al. ${ }^{[14]}$ and Hoppes et $a{ }^{[16]}$. It is evident that VV symptoms are common in middle age patients. In the present study, there were 57\% female on VV patients, so the female sex was dominated. In a study by Kamal et $a l^{[13]}$, the female sex represents $60 \%$ on visual vestibular mismatch patients. Female sex represents $70 \%$ on VV patients in a study by Dannenbaum et $a l^{[15]}$. From our study and other studies, we can conclude that $\mathrm{VV}$ is more common in female sex. Moaty et a ${ }^{[17]}$ explained that was as result of high preponderance anxiety and migraine in females rather male which are associated with VV symptoms.

The results of the present study indicate that the Arabic
VVAS had good internal consistency. Our findings are similar to those of the original study by Dannenbaum et $a l^{[15]}$, further evidencing homogeneity and good internal consistency of the AVVAS. From our study, we found some scales of VV group had the lowest corrected scales - total correlation scores, nevertheless, removal of these items did not increase Cronbach's alpha above 0.83 , so we hence agreed on keeping the AVVAS scales for the ensuing local validation of this scale. In this study, not all participants attend the follow up at audio-vestibular unit after one week; some of them filled the AVVAS again by telephone, and approximately 5-10minute needed to re- fill the scale. 7 days' time interval was chosen to minimize the effect of time on memory which might interfere with the results of the study. The study demonstrated that the AVVAS had good repeatability with high test-retest correlations $(r>0.80)$. Also the AVVAS had an acceptable stability over time. The possibility of administering the AVVAS by telephone and the small time needed to fill in the tool makes it a useful tool to screen for VV symptom among Arabic patients.

Regarding the discriminant validity, the mean score in $\mathrm{VV}$ and controls were $35.5 \pm 16.8$ versus $10.9 \pm 12.1$ respectively with significantly different $(P$ value $<0.001)$. This was similar to Dannenbaum et al study ${ }^{[15]}$, in which the mean $( \pm \mathrm{SD})$ severity score of VVAS was $30.3( \pm 26.7)$ in the VV group and $2.9( \pm 7.0)$ in the other group, further demonstrating specificity of the AVVAS. The validity and cut-off score of the AVVAS to detect abnormality depends on a comparison with normal values in the same setting in which the scale is used. From our study, the AVVAS has an ability to discriminate between controls and VV persons, also had an acceptable sensitivity, and accuracy for diagnosis VV. Validity of the AVVAS was shown by ROC curve analysis showing the appropriate cut-off value for the AVVAS was 17 . The AVVAS is highly predictive of negative outcomes

\section{CONCLUSIONS}

The AVVAS is a simple, valid and helpful screening tool to assess individuals with complaints of dizziness 
especially among Egyptian population. Further clinical Posturography should be carried out in order to compare its result with validity of the adapted Arabic VVAS. Comparisons of the Arabic $\mathrm{SCQ}^{[13]}$ and the Arabic $\mathrm{DHI}^{[12]}$ to the AVVAS's validity, reliability are recommended to study the AVVAS's sensitivity to monitor change.

\section{CONFILICT OF INTEREST}

There are no conflict of interest.

\section{REFERENCES}

1. Dimitriadis P A, Saad M, Igra M S. White matter lesions in magnetic resonance imaging of the brain in 56 patients with visual vertigo. The Journal of Laryngology and Otology 2018; 132(6): 550-553.

2. Longridge NS, Mallinson AI, Denton A. Visual vestibular mismatch in patients treated with intratympanic gentamicin for Meniere's disease. J Otolaryngol $2002 ; 31: 5-8$.

3. Furman JM, Jacob RG. A clinical taxonomy of dizziness and anxiety in the otoneurological setting. J Anxiety Disord 2001; 15:9- 26.

4. Bisdorff A, Von Brevern M, Lempert T. Classification of vestibular symptoms: towards an international classification of vestibular disorders. J Vestib Res 2009; 19:1-1.

5. Van Ombergen A, Heine L, Jillings S. Altered functional brain connectivity in patients with visually induced dizziness. NeuroImage: Clinical 2017; 14: 538-45.

6. Chin S. Visual vertigo: Vertigo of oculomotor origin. Medical hypotheses 2018; 116: 84-95.

7. Bronstein AM, et al. Vision and Vertigo: Some visual aspects of vestibular disorders. J Neurol. 2004; 251: $381-387$

8. Pavlou M., Davies R., Bronstein A. The assessment of increased sensitivity to visual stimuli in patients with chronic dizziness. J. Vestib. Res 2006; 16 (4-5), 223.
9. Agarwal K, Bronstein AM, Faldon ME. Visual dependence and BPPV. Journal of neurology. 2012; 259 (6):1117-24.

10. Nishino LK, Granato L, Campos CA, Aplicação do questionário de qualidade de vida diária em pacientes pré e pós-reabilitação vestibular. Arq. int. otorrinolaringol.(Impr.) 2008; 12(4):517-22.

11. Jafarzadeh S, Bahrami E, Pourbakht A, et al . Validity and reliability of the Persian version of the dizziness handicap inventory. Journal of research in medical sciences: the official journal of Isfahan University of Medical Sciences. 2014; 19(8): 679-775.

12. Alsanosi AA. Adaptation of the dizziness handicap inventory for use in the Arab population. Neurosciences (Riyadh). 2012; 17(2):139-44.

13. Kamal N, Taha H, El-Kabarity RH, et al. Visual vestibular mismatch: is it a vestibular disorder? Egypt J Otolaryngol 2017; 33:645.

14. Talaat HS, Moaty AS, Koura YA. Arabic version of situational characteristic questionnaire for diagnosis of visual vertigo syndrome. Hearing, Balance and Communication 2018; 23:1-7.

15. Dannenbaum E, Chilingaryan G, Fung J. Visual vertigo analogue scale: an assessment questionnaire for visual vertigo. J Vestibular Res 2011; 21: 53-159.

16. Hoppes CW, Sparto PJ, Whitney SL. cerebral activation in individuals with and without visual vertigo during optic flow: A functional near-infrared spectroscopy study. NeuroImage: Clinical. 2018; 20:655-63.

17. Moaty AS, EL, Mahallawi TH, Emara AA. The role of customized vestibular rehabilitation with visual desensitization in the management of visual vertigo syndrome. Hearing Balance and Communication. 2017;15: 127-132 